\title{
Parametric mixture models to evaluate and summarize hazard ratios in the presence of competing risks with time-dependent hazards and delayed entry
}

\author{
Bryan Lau ${ }^{\mathrm{a}}$, Stephen R. Cole ${ }^{\mathrm{b}}$, and Stephen J. Gange ${ }^{\mathrm{a}}$ \\ aDepartment of Epidemiology, Johns Hopkins Bloomberg School of Public Health, Baltimore, \\ Maryland, U.S.A.

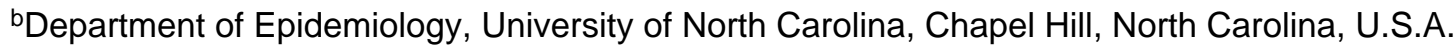

\begin{abstract}
In the analysis of survival data, there are often competing events that preclude an event of interest from occurring. Regression analysis with competing risks is typically undertaken using a causespecific proportional hazards model. However, modern alternative methods exist for the analysis of the subdistribution hazard with a corresponding subdistribution proportional hazards model. In this paper, we introduce a flexible parametric mixture model as a unifying method to obtain estimates of the cause-specific and subdistribution hazards and hazard ratio functions. We describe how these estimates can be summarized over time to give a single number that is comparable to the hazard ratio that is obtained from a corresponding cause-specific or subdistribution proportional hazards model. An application to the Women's Interagency HIV Study is provided to investigate injection drug use and the time to either the initiation of effective antiretroviral therapy, or clinical disease progression as a competing event.
\end{abstract}

\section{Keywords}

Cause-specific hazards; Competing risks; Hazard ratio; Mixture Model; Subdistribution; Subdistribution hazards; Survival analysis

\section{Introduction}

With survival data, a competing risk situation occurs when there exists an event which precludes the event of interest from occurring. The canonical example is in the analysis of specific causes of death. Death due to cancer removes the possibility of the individual experiencing death due to cardiovascular disease, for example (for reviews of competing risks, see [1]). The components of survival analysis (e.g. risk sets, hazard functions, and hazard ratios) and modeling strategies are similar but altered in construction when competing risks exist [2]. Two semi-parametric methods have become prominent approaches for competing risk analyses, a cause-specific hazard $[3,4]$ and a subdistribution hazard approach [6]. The cause-specific hazard approach and accompanying cause-specific hazards ratio $\left({ }_{c s} \mathrm{HR}\right)$ was introduced in $1978[3,4]$ and only more recently in pioneering

Copyright () 2010 John Wiley \& Sons, Ltd.

"Correspondence to: Bryan Lau, Department of Epidemiology, Johns Hopkins Bloomberg School of Public Health, 615 N. Wolfe Street, Room E7150, Baltimore, MD, 21205, U.S.A., brlau@jhsph.edu.

We declare that we have no conflict of interest. 
work by Fine and Gray [6], has the subdistribution approach for estimating the subdistribution HR $\left({ }_{s d} \mathrm{HR}\right)$ been introduced. The ${ }_{s d} \mathrm{HR}$ was motivated by the fact that the cause-specific HR $\left.{ }_{c s} \mathrm{HR}\right)$ may not reflect an association on the cumulative incidence function (CIF) [6]. Recently Beyersmann, et al. [7] illustrated a case in which the ${ }_{c s} \mathrm{HR}$ suggested a significant association, but the ${ }_{s d} \mathrm{HR}$ had a non-significant association in the opposite direction suggesting that the covariate does not affect the CIF. Beyersmann and Schumacher [8] suggested that it is for this reason that Fine and Gray's method [6] has been gaining attention in the medical literature.

An alternative to the semi-parametric methods for modeling data with competing risks is to use a parametric mixture model. Initially suggested by Cox [11], Larson and Dinse [12] expanded on the idea by decomposing the CIF into a distribution conditioned on the outcome and the probability of that outcome. Further extensions were made by Maller and Zhou [13]. Previous applications of the mixture model have focused almost exclusively on obtaining an estimate of the CIF. In previous work, the mixture model was suggested as a unifying approach between the cause-specific and subdistribution proportional hazards models, but details were not given [2]. The purpose of this paper is to give details on obtaining the ${ }_{c s} \mathrm{HR}(t)$ and ${ }_{s d} \mathrm{HR}(t)$ functions over follow-up time from a parametric mixture model that may be summarized into estimates corresponding to their proportional hazards model counterparts. While we provide an approach to summarize the ${ }_{c s} \mathrm{HR}(t)$ and ${ }_{s d} \mathrm{HR}(t)$ functions over follow-up from a parametric mixture model, it is recognized that a summary measure of the hazard ratio may have two shortcomings whether it comes from a parametric or semi-parametric model [14]. First, if the hazard ratio changes over time, then the summary association (i.e. average $\operatorname{HR}(t)$ ) that an observational study will find may be dependent on the length of follow-up. The second issue is that the $\operatorname{HR}(t)$ has a built in selection bias in that individuals at time $t$ are those remaining at risk for the outcome which may consist of those who are at less prone to develop the event [14]. Therefore, an advantage of the proposed parametric mixture model is that either the HR(t) or the summary HR for either the cause-specific or subdistribution hazards may be presented, whereas the semi-parametric proportional hazards models must either present the summary HR or be complicated by time-exposure interactions. In Section 2, we review the semi-parametric approaches and detail how the parametric mixture model can be used for hazards-ratio estimation under competing risks. In Section 3, we apply our approach to data from the Women's Interagency HIV Study consisting of a subsample of 1,164 women followed over 10 years. Finally in Section 4, we discuss the strengths and limitations of estimating either the ${ }_{c s} \mathrm{HR}$ or ${ }_{s d} \mathrm{HR}$ from a parametric mixture model.

\section{REGRESSION MODELS}

Let the observed survival data be represented by $\left(T_{i}, J_{i}\right)$ for individual $i=1, \ldots, N$ with $p \geq$ 2 types of failures, where $T_{i}$ is a non-negative random variable representing time to first event and $J_{i}$ takes a value $j$ from the set $1, \ldots, p$ to indicate the type of failure. For simplicity, we limit $p$ to the two event situation. As with most survival data, this bivariate random variable will be incomplete should the observation time end prior to any of the failure types being observed. Thus let $J_{i}=0$ when no failure of any type is observed over the period of study and $T_{i}$ is the individual's contributed time at risk. This parameterization is consistent with the two primary representations of competing risk data (e.g. as either the minimum of latent failure times [15] or as transitions to one of several absorbing states $[17,18])$. We assume that the censoring mechanism is non informative. Let the CIF be defined as $F_{j}^{*}(t)=d \operatorname{Pr}\left(T_{i} \leq t, J_{i}=j\right)$ for the $j$ th type of failure and the corresponding subdensity be defined as $f_{j}^{*}(t)=d \operatorname{Pr}\left(T_{i} \leq t, J_{i}=j\right) / d t$. Note that these are improper 
cumulative density function and density function, respectively, and therefore indicated by an “*”.

\subsection{Competing Risk Proportional Hazards Models}

Proportional hazards models using either the cause-specific hazard or the subdistribution hazard have been proposed $[3,6]$. The cause-specific hazard is defined as [3]:

$$
h_{j}(t \mid \mathbf{X})=\frac{f_{j}^{*}(t \mid \mathbf{X})}{S(t \mid \mathbf{X})}
$$

where $\mathbf{X}$ is a vector of covariates and $S(t)$ is the net survival function such that $S(t)=\operatorname{Pr}(T[G R H] t)=\exp \left(-\int_{0}^{t} \Sigma_{j=1}^{p} h_{j}(u) d u\right)=\exp \left(-\int_{0}^{t} h(u) d u\right)$ in which $h(t)$ is the net hazard function for having any of the competing events. This can be used to construct a cause-specific proportional hazards model [3]:

$$
h_{j}(t \mid \mathbf{X})=h_{0 j}(t) \exp \left(\mathbf{X}^{\mathrm{T}} \beta_{j}\right) \quad j=1,2
$$

where $h_{0 j}(t)$ is an arbitrary baseline cause-specific hazard function and $\beta_{j}$ is the vector of unknown coe cents for covariates $\mathbf{X}$. As previously stated, a difficulty of the cause-specific proportional hazards model is that the interpretation of $\exp \left(\beta_{j}\right)$ as the hazards ratio does not necessarily translate into an effect on the cumulative event probabilities since the CIF is a

function of the hazards from all events that is $F_{j}^{*}(t)=\int_{0}^{t} S(u-) h(u) d u$ is dependent on all events due to the net survival function $S(t)[6,2,7]$. However, it is important to note that the hazard ratio is still interpretable as a measure of association in terms of the cause-specific hazards.

Alternatively, the subdistribution hazard may be used directly to construct a different proportional hazards model. The subdistribution hazard is defined as $[5,6]$ :

$$
\begin{aligned}
\lambda_{j}(t \mid \mathbf{X}) & =\frac{f_{j}^{*}(t \mid \mathbf{X})}{1-F_{j}^{*}(t \mid \mathbf{X})} \\
& =\frac{f_{j}^{*}(t \mid \mathbf{X})}{\operatorname{Pr}(J \neq j \mid \mathbf{X})+S_{j}^{*}(t \mid \mathbf{X})}
\end{aligned}
$$

where $S_{j}^{*}(t)=\operatorname{Pr}(J=j)-F_{j}^{*}(t)$ is the subsurvivorship function. The corresponding proportional hazards model is:

$$
\lambda_{j}(t \mid \mathbf{X})=\lambda_{0 j}(t) \exp \left(\mathbf{X}^{\mathrm{T}} \phi_{j}\right) \quad j=1,2
$$

where $\lambda_{0 j}(t)$ is an arbitrary baseline subdistribution hazard function and $\varphi_{j}$ is the vector of unknown coefficients for covariates $\mathbf{X}$. The subdistribution hazard ratio, $\exp \left(\varphi_{j}\right)$, from a subdistribution proportional hazards model may be directly interpreted as an effect upon the CIF. It should be noted for the Fine and Gray subdistribution proportional hazards model that if the subdistribution proportional hazards model is assumed to be proportional for event 1 then assuming a subdistribution proportional hazards model for event 2 is misspecified $[9,10]$. Furthermore, the relationship between cause-specific hazards and subdsitribution hazards implies that if proportionality assumption of one type of hazard is met then the other 
will not be proportional [8]. Therefore as explored elsewhere [7,8,19], $\beta_{j}$ does not generally equal $\varphi_{j}$.

\subsection{Parametric Mixture Model}

Larson and Dinse [12] describe an alternative competing risks model represented by a parametric mixture model rather than the semi-parametric proportional hazards model. The CIF corresponding to the $j$ th type of failure is decomposed as:

$$
\begin{aligned}
F_{j}^{*}(t \mid \mathbf{X}) & =\operatorname{Pr}\left(T \leq t \mid J=j, \mathbf{X}_{\mathbf{j}}\right) \operatorname{Pr}\left(J=j \mid \mathbf{X}_{\omega}\right) \\
& =F_{j}\left(t \mid \mathbf{X}_{j}\right) \operatorname{Pr}\left(J=j \mid \mathbf{X}_{\omega}\right)
\end{aligned}
$$

with the corresponding subdensity function defined as:

$$
\begin{aligned}
f_{j}^{*}(t \mid \mathbf{X}) & =\frac{d \operatorname{Pr}\left(T \leq t \mid J_{i}=J, \mathbf{X}_{\mathrm{j}}\right)}{d t} \operatorname{Pr}\left(J=j \mid \mathbf{X}_{\omega}\right) \\
& =f_{j}\left(t \mid \mathbf{X}_{\mathrm{j}}\right) \operatorname{Pr}\left(J=j \mid \mathbf{X}_{\omega}\right) .
\end{aligned}
$$

where $\mathbf{X}_{\mathbf{j}} \subseteq \mathbf{X}$ and $\mathbf{X}_{\omega} \subseteq \mathbf{X}$. The distribution functions $F_{j}(t)$ and $f_{j}(t)$ are modeled by an appropriate distribution of choice. For the situation of two competing events, the mixture parameter $\operatorname{Pr}\left(J=j \mid \mathbf{X}_{\omega}\right)$ is modeled using a binary data model (e.g. logistic) for the failure types. These building blocks can be used to construct a likelihood for the $i$ th individual's contribution when there are two competing outcomes measured from a common origin such that:

$$
L_{i}=\left[\pi_{i} f_{1}\left(t_{i} \mid \mathbf{X}_{\mathrm{i} 1}\right)\right]^{\delta_{i}} \times\left[\left(1-\pi_{i}\right) f_{2}\left(t_{i} \mid \mathbf{X}_{\mathbf{i} 2}\right)\right]^{\theta_{i}} \times\left[\pi_{i} S_{1}\left(t_{i} \mid \mathbf{X}_{\mathrm{i} 1}\right)+\left(1-\pi_{i}\right) S_{2}\left(t_{i} \mid \mathbf{X}_{\mathbf{i} 2}\right)\right]^{\left(1-\delta_{i}-\theta_{i}\right)}
$$

where $\mathbf{X}_{\mathrm{i} 1}$ and $\mathbf{X}_{\mathrm{i} 2}$ are the $i$ th individual's corresponding set of covariates being modeled for the events $J=1$ and $J=2$ respectively; $t_{i}$ is the $i$ th individual's observed time until the first of the two outcomes as reflected by the indicator functions:

$$
\delta_{i}=\left\{\begin{array}{ccc}
0 & \text { if } & J_{i} \neq 1 \\
1 & \text { if } & J_{i}=1
\end{array} \text { and } \theta_{i}=\left\{\begin{array}{ccc}
0 & \text { if } & J_{i} \neq 2 \\
1 & \text { if } & J_{i}=2
\end{array}\right.\right.
$$

such that individuals are right censored if $\delta_{i}=\theta_{i}=0 ; \pi_{i}=\operatorname{Pr}\left(J_{i}=1 \mid \mathbf{X}_{\omega}\right)$ are the mixture probabilities; $f_{1}\left(t_{i} \mid \mathbf{X}_{\mathrm{i} 1}\right)$ and $f_{2}\left(t_{i} \mid \mathbf{X}_{\mathrm{i} 2}\right)$ are the probability density functions for the parametric distribution being used for two competing events and similarly $S_{1}\left(t_{i} \mid \mathbf{X}_{\mathrm{i} 1}\right)$ and $S_{2}\left(t_{i} \mid \mathbf{X}_{\mathrm{i} 2}\right)$ are the corresponding survivor functions. Extensions of (5) to $p>2$ is straightforward.

Since $f_{j}^{*}(t), F_{j}^{*}(t)$, and the net survivorship function $\left(S(t)=\pi\left(S_{1}(t)\right)+(1-\pi) S_{2}(t)\right)$ are determined by the parameter estimates, it is easy to see that the cause-specific hazard

functions $\left(f_{j}^{*}(t) / S(t)\right)$ and the subdistribution hazard functions $\left(f_{j}^{*}(t) /\left[1-F_{j}^{*}(t)\right]\right)$ can also be determined. These building blocks allow for the estimation of the ratio of cause-specific hazards, as a function of time $t$ :

$$
\begin{aligned}
{ }_{c s} \mathrm{HR}_{j}(t \mid X) & =\frac{h_{j}(t \mid \mathbf{X})}{h_{0 j}(t)} \\
& =\frac{f_{j}^{*}(t \mid \mathbf{X})}{S_{j}(t \mid \mathbf{X})} / \frac{f_{0 j}^{*}(t)}{S_{0 j}(t)}
\end{aligned}
$$


where $f_{0 j}^{*}(t)$ is the corresponding subdensity function for the arbitrary baseline hazard function and $S_{0 j}(t)$ is the net survival function for individuals with the covariate values that correspond to the reference population that defines the baseline arbitrary hazard function. Similarly the subdistribution hazard ratio curves can be estimated as:

$$
\begin{aligned}
{ }_{s d} \operatorname{HR}_{j}(t \mid X) & =\frac{\lambda_{j}(t \mid \mathbf{X})}{\lambda_{0 j}(t)} \\
& =\frac{f_{j}^{*}(t \mid \mathbf{X})}{1-F_{j}(t \mid \mathbf{X})} / \frac{f_{0 j}^{*}(t)}{1-F_{0 j}^{*}(t)}
\end{aligned}
$$

where $f_{0 j}^{*}(t)$ and $F_{0 j}^{*}(t)$ are the corresponding subdensity function and CIF for the arbitrary baseline hazard function, respectively.

The likelihood function in (5) can be modified to allow for left truncation (Appendix). This would allow estimation of the $\operatorname{HR}(t)$ while avoiding bias due to late entry into the study or for incorporating time-varying covarites into the survival models [15]. While it is straight forward to incorporate left truncation into the cause-specific proportional hazards model, it is not easily done for the subdistribution proportional hazards model [16]. Additionally uncertainty in exact timing of the event could be incorporated through interval censoring (Appendix), which is not easily accounted for in the semi-parametric proportional hazards models.

To facilitate appropriate inferences, the parametric distributions to be included in the mixture model need to accurately reflect the data. To estimate $f_{1}\left(t_{i}\right)$ and $f_{2}\left(t_{i}\right)$ and their respective survivor functions, we propose using a flexible parametric distribution. The three parameter generalized gamma distribution $\operatorname{GG}(\beta, \sigma, \lambda)$ is one option in which the hazard can take various shapes including declining, increasing, bathtub shape (decreasing then increasing), or arc shaped (increasing then decreasing) hazards [20]. In fact, the generalized gamma distribution can accommodate a whole range of forms including many of the most commonly used distributions, such as exponential, gamma, log-normal, and Weibull distributions as special cases [20]. Another broad class of models that could be utilized is the generalized $\mathrm{F}$ family [21]. Maximum likelihood estimation of the parameters for the mixture model can be performed in SAS using the NLMIXED procedure. This procedure allows for the computation of general functions of the parameter estimates using the estimate statement including estimates of the hazard and hazard-ratio functions. Standard errors for these functions can be determined using standard likelihood inferrence techniques (e.g. Waldbased standard errors calculated using the delta method). However for some non-linear models these standard errors may not adequately reflect the true variance and we would recommend using either the profile likelihood [22] or bootstrap methods [23,24].

2.2.1. Summarizing the $\mathbf{H R}(\mathbf{t})$ Curve-The above approach provides an evaluation of how exposures impact the hazard rates of different competing events over time. In many settings, it may be desirable to summarize these hazards over follow-up and provide a single summary estimate comparable to the HR typically obtained from a proportional hazards model. This is often the case in the medical setting in which clinicians want to quickly disseminate information regarding the relationship between an exposure and outcome. A single number is much easier to convey compared to either two hazard functions or a HR curve in which each is a function of time. The summary HR from a proportional hazards model where the proportionality assumption is unmet essentially weights the ratio of the time-dependent hazards at each failure time-point equivalently assuming few or no tied events $[25,26]$. Such weighting may not be the most appropriate in summarizing the timedependent hazard ratios [26,27,28,29]. Briefly, Kalbfleisch and Prentice [27] introduced a 
summary measure of the hazard ratio when proportionality was not met by allowing the hazards to be weighted by some survivor function, $G_{\alpha}(t)=S_{0}^{\alpha}(t) S_{1}^{\alpha}(t)$ for $(t>0)$ in which $S_{0}(t)$ and $S_{1}(t)$ are the survivor functions for the two sample problem and $\alpha>0$ such that $\alpha=$ 0.5 would be the geometric average of the two survivor functions and $\alpha<.5$ or $\alpha>.5$ would emphasize earlier or later periods, respectively [27]. Additional work in this area has been done providing potentially other metrics, e.g. weighted by number at risk providing a Wilcoxon-type estimator $[26,28,29]$. However, Grambauer et al. have shown that the subdistribution hazard ratio when mis-specified is still useful as it is the least false parameter which may be interpreted as the time-averaged effect on the cumulative event probabilities [10]. Nevertheless, we present here a simple outline in which all points are equivalently weighted. However, our methods may be appropriately adapted to include such alternative weights. We outline two methods. The first estimates the hazard curves for an "average" individual with and without exposure. The ratio of these cuves are then determined at each of the $w$ unique failure time points.

- "Average" individual approach (Method 1)

1. Create a record with the mean covariate level for each covariate such that the record has a covariate vector of means $\bar{X}=1 / N \Sigma_{i=1}^{N} \mathbf{X}$.

2. Let $w=w_{1}, \ldots, w_{K}$ correspond to the ordered unique failure times, $T$ such that $w_{1}=$ $\min \left(T_{1}, \ldots, T_{N}\right)$ and $w_{K}=\max \left(T_{1}, \ldots, T_{N}\right)$.

3. Duplicate the mean covariate record and assign the exposure variable to be "unexposed" to the original record and the exposure variable as "exposed" for the duplicate record. Should the exposure of interest be continuous then the difference between exposed and unexposed values should be 1 unit of scientific interest centered around the study population mean.

4. Duplicate the pair of records $K$ times giving the $k$ th replicate the $w_{k}$ failure time.

5. Estimate either the cause-specific or subdistribution hazard for each of the $2 W$ records and obtain $\operatorname{HR}\left(t=w_{k}\right)=g_{1}(t) / g_{0}(t)$ where $g_{1}(t)$ and $g_{0}(t)$ correspond to the exposed and unexposed hazard functions, respectively.

6. The mean of these time-specific HRs (e.g. $\left.\overline{\mathrm{HR}}=1 / K \Sigma_{w=w_{1}}^{w_{K}} \mathrm{HR}(t=w)\right)$ corresponds to the ${ }_{c s} \mathrm{HR}$ or ${ }_{s d} \mathrm{HR}$ obtained from the proportional hazards models depending on whether $g(t)$ is the cause-specific or subdistribution hazard function, respectively.

7. Bootstrap methods $[23,24]$ may be applied to estimate the uncertainty in this summary HR.

This approach is easily accomplished in SAS by creating the average unexposed and exposed records and appending this to the original data file. These additional records can be excluded from the fitting process by utilizing the replicate statement with a weight variable to identify the original records from the created records. Additional programming statements to the NLMIXED procedure are then added to estimate the both the cause-specific and subdistribution hazards and the predict statement is used to output each of the hazards.

The second method entails generating two hazard curves for each individual (one for the observed exposure status, and one for the unobserved exposure status).

- Empirically weighted approach (Method 2)

1. Let $w=w_{1}, \ldots, w_{K}$ correspond to the ordered unique failure times, $T$ such that $w_{1}=$ $\min \left(T_{1}, \ldots, T_{N}\right)$ and $w_{K}=\max \left(T_{1}, \ldots, T_{N}\right)$. 
2. For each of the $N$ individuals, create a duplicate record that matches the original on all covariates with the exception of the variable of interest. Instead, the duplicate record is given the unobserved (i.e., opposite) exposure value.

3. For each $N$ pairs of records, replicate the pair $K$ times giving the $k$ th pair of records the $w_{k}$ failure time. Therefore the final database will have $2 * N * K$ records.

4. Estimate either the cause-specific or subdistribution hazards at the corresponding failure time for each of the records by using the estimated model parameters and the cause-specific hazard function $\left(f_{j}^{*}(t) / S(t)\right)$ and subdistribution hazard function $\left(f_{j}^{*}(t) /\left[1-F_{j}^{*}(t)\right]\right)$. Thus the $\operatorname{HR}_{i}(t=w)=g_{1 i}(t) / g_{0 i}(t)$ where $g_{1 i}(t)$ and $g_{0 i}(t)$ correspond to the exposed and unexposed hazard functions, respectively, for the $i$ th individual at the $w$ failure time.

5. Then the $\overline{\mathrm{HR}}=1 /(N K) \Sigma_{i=1}^{N} \Sigma_{w=w_{1}}^{w_{K}} \mathrm{HR}_{i}(t=w)$ corresponds to either the ${ }_{c s} \mathrm{HR}$ and ${ }_{s d} \mathrm{HR}$ depending on whether $g(t)$ is the cause-specific or subdistribution hazard function, respectively.

6. Bootstrap methods $[23,24]$ may be applied to estimate the uncertainty in this summary HR.

\section{APPLICATION TO DATA FROM THE WOMEN'S INTERAGENCY HIV STUDY}

We applied these methods to data from the Women's Interagency HIV Study (henceforth, the Study) to examine whether women with a history of injection drug use were more or less likely to have one of two competing events: either initiating highly active antiretroviral therapy (henceforth, therapy) or the onset of clinical disease progression as defined by the occurrence of either an AIDS defining illness or death [2]. Briefly, the Study was established in August of 1993 to comprehensively examine the impact of HIV on women at six US sites [30]. For this application, we are interested in whether individuals with a history of injection drug use were less likely to initiate therapy prior to AIDS or death as compared to those without a history of injection drug use. Prior studies have shown that HIV-infected individuals with past injection drug use are less likely to initiate therapy $[31,32,33,34]$ and are more likely to die in the era of effective treatment for HIV $[34,35,36]$. Yet the comparison of treatment initiation prior to disease progression (defined by AIDS or death) has not been well explored in a competing risk framework.

The study sample consisted of 1,164 women that were alive and free of clinical AIDS as of December 6, 1995, when the first protease inhibitor (Saquinavir mesylate) was approved by the US Federal Drug Administration. This date marks the first time-point in which individuals could be placed on a highly active antiretoviral drug regimen. Women were followed until the first of: initiation of therapy, an AIDS diagnosis, death, or administrative censoring defined as September 28, 2006. Covariates included history of injection drug use upon study enrollment, whether an individual was African-American, age on December 6 1995, and CD4 nadir prior to December 61995.

The study sample consisted of 439 women with and 725 without a history of injection drug use. Overall, 679 (58\%) women initiated therapy prior to either AIDS or death; 359 (31\%) had the competing event and either died or had an AIDS diagnosis before initiation of effective therapy. The remaining $11 \%$ were censored (5\% loss to follow-up, $5 \%$ administratively censored). Reflecting the demographics of HIV in women and in the cohort, the majority of the study population was African-American (58\%) and had a median 
age of 36 (interquartile range 31-41) as of December 6, 1995. A greater proportion of injection drug users tended to be African-American and of older age ( $\mathrm{p}=0.017$ and $p<$ 0.001 , respectively).

The maximum likelihood estimates for the mixture model are shown in Table 1. A lognormal distribution was used to model initiation of therapy $\left(f_{1}\right)$ as a sufficiently small enough gradient could not be achieved for the shape parameter when modeling a generalized gamma distribution. However, the shape parameter indicated that the distribution was tending towards a lognormal distribution which is a limiting case for the generalized gamma distribution [20,37]. A generalized gamma distribution was used to model the incidence of AIDS/Death prior to therapy $\left(f_{2}\right)$. Under this model, an African-American woman with a history of injection drug use that is 36 years of age with a CD4 nadir of $349 \mathrm{cells} / \mathrm{mm}^{3}$ has a probability of 0.46 for ever initiating therapy $\left(\pi_{i}=e^{1.0754-0.3222-.8976} /(1+\right.$ $\left.e^{1.0754-0.3222-.8976}\right)=0.46$ ). The CIF estimated from the mixture model as compared to a non-parametric cumulative incidence function estimator [4] is shown in Figure 1. The nonparametric cumulative incidence function estimator was weighted with inverse probability weights for history of injection drug use in order to provide curves adjusted for race, age, and CD4 nadir on December 61995 [38,39] that would be comparable to the adjusted parametric curves by injection drug use status. Additionally both the cause-specific hazard and subdistribution hazard curves are shown along with the corresponding hazard ratios. Given that the generalized gamma distribution for the initiation of therapy tended towards a lognormal distribution, which was used in the final model, this suggests that a proportional hazards model might not be appropriate. This is apparent in Figure 1 as the hazard curves are not uniformly equidistant for both the initiation of therapy and for the subdistribution hazards estimates for AIDS/Death prior to therapy. For example the ${ }_{s d} \mathrm{HR}$ for initiation of therapy is generally increasing up to 1 year and then subsequently decreasing. However while the number of events are increasing prior to 1 year as shown by the histogram in Figure 1e, there are not many events perhaps suggesting that one could discount the initial increase in ${ }_{s d} \mathrm{HR}$. Neverthless, the subsequent decrease between 1 and 5 years is within the bulk of when the majority of events are occuring. This suggests that with increased amount of time from 6 December 1995 when effective therapy first became available, individuals with a history of injection drug use were even less likely to initiate prior to disease progression to AIDS or death than those without a history of drug use. Conversely the ${ }_{s d} \mathrm{HR}$ for disease progression (Figure 1f) before therapy initiation shows an increasing ${ }_{s d} \mathrm{HR}$ between 1 and 5 years for those with a history of injection drug use as compared to those without.

The Cox proportional hazards model (and similarly the subdistribution proportional hazards model) is often used in research despite potential violation of the proportional hazards assumption. While the subdistribution proportional hazards model still provides the least false parameter when mis-specified $[19,10]$, the summary hazard ratios from any proportional hazards model still has shortcomings such as the dependency on total length of follow-up and the potential for selection bias [14]. Despite concerns about the appropriateness of the proportional hazards assumption (global test for cause-specific proportional hazards assumption, therapy initiation: $\mathrm{p}<0.001$; AIDS/Death: $\mathrm{p}=0.29$, subdistribution proportional hazards assumption, therapy initiation: $\mathrm{p}<0.001$; AIDS/Death: $\mathbf{p}<0.001$ ), we constructed both cause-specific and subdistribution hazard ratios (Table 2). Both the ${ }_{c s} \mathrm{HR}$ and ${ }_{s d} \mathrm{HR}$ their $95 \%$ confidence intervals (obtained from bootstrap methods with 1000 replicates) were similar between the summarized mixture estimates and proportional hazards models. To obtain the bootstrap confidence intervals individuals were resampled all 1164 women with replacement and the model was re-estimated based upon each sample. The 0.025 and 0.975 quantiles of the HR estimates were then obtained to provide the confidence intervals. These results show that the parametric mixture model 
allows for the estimation of both the summarized cause-specific and subdistribution HR that is equivalent to the semi-parametric proportional hazards models. However, an advantage of the parametric mixture model over the semi-parametric approaches is the ablity to handle left truncation (subdistribution proportional hazards model [16]) and interval censoring (both proportional hazards models) easily. Furthermore, both semi-parametric models may fail to meet the proportional hazards assumption and the assumption cannot hold simultaneously for both types of proportional hazards models [19,8]. Figure 2 shows the results in which age is made the time scale for the analysis and therefore requires left truncation. The model is adjusted for CD4 nadir, race, and time on study. The ${ }_{c s} \mathrm{HR}(t)$ and ${ }_{s d} \mathrm{HR}(t)$ are not proportional as evident by the non-constant HR over the entire range of ages. While a standard cause-specific proportional hazards could still be used, the interpretation would be complicated by the inclusion of time-interactions. Alternatively the average ${ }_{c s} \mathrm{HR}$ could be calculated using an appropriate weighting scheme [26,27,28]. However, note that while this may be appropriate for initiation of therapy it appears less appropriate for progression to AIDS or death as the $\operatorname{HR}(t)$ is qualitatively different before $(\mathrm{HR}>1)$ and after $(\mathrm{HR}<1)$ age 47 . This qualitative change in ${ }_{c s} \mathrm{HR}$ for AIDS/Death such that history of injection drug use is protective at older ages unlikely to reflect a true protective effect (figure $2 b$ ), but is more likely to be a reduction in the number of susceptible individuals among those with history of injection drug use at least relative to those without a history of injection drug use. This illustrates the potential for selection bias in the HR [14]. Indeed the ${ }_{s d} \mathrm{HR}$ remains elevated and increasing, indicating that the cumulative probability for disease progression to AIDS/death prior to therapy initiation remains higher among those with a history of injection drug use (Figure 2d).

\section{DISCUSSION}

The cause-specific proportional hazards model for competing risk analysis has been used since the 1980s [3,4]. Only with the relatively recent introduction of the subdistribution proportional hazards model by Fine and Gray [6] have regression methods evolved to estimate a hazard ratio that is directly interpretable with the cumulative incidence function (which may be estimable with left truncated data in the R package etm [41]). Several recent papers have discussed the effect of a covariate on either type of hazard may be quite different $[7,8,19]$. Our work demonstrates how a unifying parametric mixture model can be used to estimate both the cause-specific or subdistribution hazard functions and the corresponding ${ }_{c s} \mathrm{HR}$ and ${ }_{s d} \mathrm{HR}$. Our example illustrates that the proposed parametric method may result in similar inferences and $95 \%$ confidence intervals. There are several advantages of the parametric mixture model over the semi-parametric approaches. First the parametric approach is not limited by the strong proportional hazards assumption. While this can be overcome by inclusion of time-covariate interactions this can complicate the interpretation. While incorporation of appropriate weights at each failure time may alleviate some of the interpretation difficulties in obtaining an average HR [26,27,28,29], this approach may obscure qualitative differences in the $\operatorname{HR}(t)$. Second, the parametric mixture model allows for left truncation for estimating the ${ }_{s d} \mathrm{HR}(t)$, while current semi-parametric methods for estimating the ${ }_{s d} \mathrm{HR}(t)$ cannot handle left truncation. Finally, an advantage of all parametric methods over semi- and non-parametric approaches is that if an appropriate distribution has been chosen then parametric methods will be statistically more efficient.

Two methods for summarizing the hazard curves were presented. The "average" individual approach is convenient in that it is less computationally intensive. However, it can be counterintuitive to estimate the hazard function the "average covariate values" when covariates are binary (e.g. sex), substituting the mean value may not be consistent with the meaning of the data. Therefore, while the averages for each covariate are used, there is no guarantee that these are attained by any individual. The second empirically weighted 
approach uses the original data as it occurred with the alternative level of exposure. It overcomes the disadvantages of the average approach but requires more time in the construction of the estimate, with the replication of each individual to have a set of failure time points with and without exposure. However should the proportional hazard assumption be violated, it is preferable to present the hazard functions and HR curves as funtions of time rather than the summarized estimate. This will then convey information about how the association between exposure and outcome changes over time similar to methods we have previously proposed comparing two competing events [40]. The results from the semiparametric and mixture model approaches applied to the data from the Women's Interagency HIV Study yielded similar results. This is as one would expect given that a proportional hazards model essentially weights the ratio of the time-dependent hazards at each failure time-point equivalently and the mixture model was also similarly weighted. However, besides the advantage of providing the $\operatorname{HR}(t)$, the mixture model approach can easily be adapted to include a more appropriate weighting scheme that would provide a different summary result than the standard proportional hazards model $[26,27,28,29]$.

The mixture model approach is not the only parametric method for competing risks in the literature. Jeong and Fine $[42,43]$ provided a method that relies on the Gompertz distribution to directly parametrize the cumulative incidence function. The Gompertz distribution was chosen as it is an improper distribution and therefore a natural distribution for modeling the cumulative incidence function and may or may not provide a better fit than the mixture model [42]. However, as the authors state, the two-parameter Gompertz distribution only allows monotone hazard shapes. In addition, the cause-specific hazards are not estimable.

Furthermore, like many survival analysis methods, the mixture model presented assumes that eventually one of the competing events will occur (i.e. $\Sigma_{j=1}^{p} \operatorname{Pr}(J=j)=1$ ). This may be a reasonable assumption for some situations (e.g. where all cause mortality is a competing

event), but this assumption may be inappropriate in other cases in which $\Sigma_{j=1}^{p} \operatorname{Pr}(J=j) m v g 1$ indicating that some individuals are immune to the risks under investigation. In this situation, Maller and Zhou [13] have extended the mixture model to situations in which there may be individuals "immune" to the outcomes and may be of interest. Furthermore, a concern with all parametric methods is whether the chosen distribution is appropriate to describe the data. The use of a mixture of generalized gamma distributions allows the model to be flexible and includes some common distributions (such as exponential, log-normal, and Weibull distributions) as special cases of the generalized gamma family [20].

Additionally, because of the parametric nature of the model one can extrapolate beyond the point in which there is no additional data. However, one must be cautious in doing so to avoid over interpreting or over extending the inferences. Neverthless, semi-parametric and non-parametric methods are not able to do this. Moreover, the mixture model may be less robust than the semi-parametric proportional hazards model counterparts. This may be the case in which there are only a small number of the two competing events in which the addition of a few more of either of the events may change the inferences from the model. Of course this should not be a problem if there are a substantial number of events.

In conclusion, we have extended the use of a mixture model for competing risks. The mixture model provides a unifying approach to estimate both the cause-specific and subdistribution hazard functions as well as a summary estimate of the ${ }_{c s} \mathrm{HR}$ and ${ }_{s d} \mathrm{HR}$. Estimates of the latter two are comparable to those obtained from proportional hazards models based upon either the cause-specific or subdistribution hazards. However, the mixture model has the advantage of being able to be flexible and provide an estimate the hazard ratios as a function of time or as a summary measure regardless of whether or not left truncation or interval censoring is present in the data. 


\section{Acknowledgments}

We receive funding from the National Institutes of Health (U01-AI-42590 for the Women's Interagency HIV Study; U01-AI069918 for the North American AIDS Cohort Collaboration on Research and Design, which is a part of the International Epidemiologic Databases to Evaluate AIDS (IeDEA); and K01-AI071754 (to B.L.)). The funding sources have had no involvement with this manuscript.

Contract/grant sponsor: National Institutes of Health (U01-AI-42590, U01-AI069918, K01-AI071754)

\section{APPENDIX}

As previously stated, the likelihood function in equation (5) can be modified to allow for left truncation in order to allow estimation of the $\operatorname{HR}(t)$ while avoiding bias due to late entry into the study or for incorporating time-varying covariates into the survival models. We have discarded the covariate vector, $\mathbf{X}_{\mathrm{i} 1}$ and $\mathbf{X}_{\mathrm{i} 2}$, from the equations to simplify the notation.

$$
L_{i}=\left[\pi_{i} \frac{f_{1}\left(t_{i}\right)}{S_{1}\left(v_{i}\right)}\right]^{\delta_{i}} \times\left\lceil\left(1-\pi_{i}\right) \frac{f_{2}\left(t_{i}\right)}{S_{2}\left(v_{i}\right)}\right]^{\theta_{i}} \times\left[\pi_{i} \frac{S_{1}\left(t_{i}\right)}{S_{1}\left(v_{i}\right)} \times\left(1-\pi_{i}\right) \frac{S_{2}\left(t_{i}\right)}{S_{2}\left(v_{i}\right)}\right]^{\left(1-\delta_{i}-\theta_{i}\right)}
$$

where $v_{i}>0$ and $v_{i}=0$ for the $i$ th individuals with and without truncated times, respectively. Therefore such individuals are observed from $v_{i}$ to $t_{i}$. Additionally the likelihood function could be further modified to allow for interval censoring which would be difficult to incorporate in either the cause-specific or subdistribution proportional hazards models.

$$
\begin{aligned}
L_{i}=\left[\pi_{i} \frac{f_{1}\left(t_{i}\right)}{S_{1}\left(v_{i}\right)}\right]^{\delta_{i}\left(1-\zeta_{i}\right)} \\
\times\left[\pi_{i} \frac{S_{1}\left(t_{2 i}\right)-S_{1}\left(t_{1 i}\right)}{S_{1}\left(v_{i}\right)}\right]^{\left(\delta_{i} \zeta_{i}\right)} \\
\times\left[\left(1-\pi_{i}\right) \frac{f_{2}\left(t_{i}\right)}{S_{2}\left(v_{i}\right)}\right]^{\theta_{i}\left(1-\eta_{i}\right)} \\
\times\left[\left(1-\pi_{i}\right) \frac{S_{2}\left(t_{2 i}\right)-S_{2}\left(t_{1 i}\right)}{S_{2}\left(v_{i}\right)}\right]^{\left(\theta_{i} \eta_{i}\right)} \\
\times\left[\pi_{i} \frac{S_{1}\left(t_{i}\right)}{S_{1}\left(v_{i}\right)} \times\left(1-\pi_{i}\right) \frac{S_{2}\left(t_{i}\right)}{S_{2}\left(v_{i}\right)}\right]^{\left(1-\delta_{i}-\theta_{i}\right)}
\end{aligned}
$$

where $t_{1 i}<t_{2 i}$ and $t_{1 i}$ and $t_{2 i}$ indicate the two boundary times of the interval in which the event $J_{i}=1$ or $J_{i}=2$ occurs, $\zeta_{i}$ is an indicator function for interval censoring for the $J_{i}=1$ event, and $\eta_{i}=1$ is an indicator function for identifying individuals who are interval censored for the $J=2$ event within the $t_{1 i}$ to $t_{2 i}$ interval.

\section{References}

1. Putter H, Fiocco M, Geskus RB. Tutorial in biostatistics: Competing risks and multi-state models. Statistics in Medicine. 2007; 26:2389-2430. DOI: 10.1002/sim.2712. [PubMed: 17031868]

2. Lau B, Cole SR, Gange SJ. Competing risk regression methods for epidemiologic data. American Journal of Epidemiology. 2009; 170:244-256. DOI:10.1093/aje/kwp107. [PubMed: 19494242]

3. Prentice RL, Kalbleisch JD, Peterson AV Jr. et al. The analysis of failure times in the presence of competing risks. Biometrics. 1978; 34:541-554. [PubMed: 373811]

4. Kalbfleisch, JD.; Prentice, RL. The Statistical Analysis of Failure Time Data. John Wiley and Sons, Inc.; New York, NY: 1980. p. 247-277. 
5. Gray RJ. A class of K-sample tests for comparing the cummulative incidence of a competing risk. The Annals of Statistics. 1988; 16:1141-1154.

6. Fine JP, Gray RJ. A Proportional Hazards Model for the Subdistribution of a Competing Risk. Journal of the American Statistical Association. 1999; 94:496-509.

7. Beyersmann J, Dettenkofer M, Bertz H, et al. A competing risks analysis of bloodstream infection after stem-cell transplantation using subdistribution hazards and cause-specific hazards. Statistics in Medicine. 2007; 26:5360-5369. DOI: 10.1002/sim.3006. [PubMed: 17634974]

8. Beyersmann J, Schumacher M. Letter to the editor: Misspecificed regression model for the subdistribution hazard of a competing risks. Statistics in Medicine. 2007; 26:1649-1652. DOI: 10.1002/sim.2727. [PubMed: 17054104]

9. Beyersmannn J, Latouche A, Buchholz A, Schumacher M. Simulating competing risks data in survival analysis. Statistics in Medicine. 2009; 28:956-971. DOI: 10.1002/sim.3516. [PubMed: 19125387]

10. Grambauer N, Schumacher M, Beyersmann J. Proportional subdistribution hazards modeling offers a summary analysis, even if misspecified. Statistics in Medicine. 2010; 29:875-884. DOI: 10.1002/sim.3786. [PubMed: 20213713]

11. Cox DR. The Analysis of Exponentially Distributed Lifetimes with 2 Types of Failure. Journal of the Royal Statistical Society Series B-Statistical Methodology. 1959; 21:411-21.

12. Larson MG, Dinse GE. A mixture model for the regression analysis of competing risks data. Applied Statistics. 1985; 34:201-211.

13. Maller RA, Zhou X. Analysis of parametric models for competing risks. Statistica Sinica. 2002; 12:725-750.

14. Hernán M. The Hazards of Hazard Ratios. Epidemiology. 2010; 21:13-15. DOI:10.1097/EDE. 0b013e3181c1ea43. [PubMed: 20010207]

15. Klein, JP.; Moeschberger, ML. Survival Analysis: Techniques for Censored and Truncated Data. 2nd edition. Springer Verlag; New York, NY: 2003.

16. Geskus RB. Cause-Specific Cumulative Incidence Estimation and the Fine and Gray Model Inder both Left Truncation and Right Censoring. Biometrics. 2010 in press. DOI: 10.1111/j. 1541-0420.2010.01420.x.

17. Klein JP, Andersen PK. Regression Modeling of Competing Risks Data based on Pseudovalues of the Cumulative Incidence Function. Biometrics. 2005; 61:223-229. DOI: 10.1111/j.0006-341X. 2005.031209.x. [PubMed: 15737097]

18. Andersen PK, Abildstrom SZ, Rosthøj S. Competing risks as a multi-state model. Statistical Methods in Medical Research. 2002; 11:203-215. DOI: 10.1191/0962280202sm281ra. [PubMed: 12040697]

19. Latouche A, Boisson V, Chevret S, Porcher R. Misspecified regression model for the subdistribution hazard of a competing risk. Statistics in Medicine. 2007; 26:965-974. DOI: 10.1002/sim.2600. [PubMed: 16755533]

20. Cox C, Chu H, Schneider MF, Muñoz A. Parametric survival analysis and taxonomy of hazard functions for the generalized gamma distribution. Statistics in Medicine. 2007; 26:4352-4374. DOI: 10.1002/sim.2836. [PubMed: 17342754]

21. Cox C. The generalized F distribution: an umbrella for parametric survival analysis. Statistics in Medicine. 2008; 27:4301-4312. DOI: 10.1002/sim.3292. [PubMed: 18407568]

22. Bates, DM.; Watts, DG. Nonlinear Regression Analysis and Its Applications. Wiley; New York, NY: 1998.

23. Efron, B. The Jacknife, the Bootstrap, and Other Resampling Plans. Society for Indiustrial and Applied Mathmatics; Philadelphia, PA: 1982.

24. Efron, B.; Tibshirani, R. An introduction to the Bootstrap. Chapman Hall; London: 1993.

25. Lagakos SW, Schoenfeld DA. Properties of Propostional-Hazards Score Tests under Misspecified Regression Models. Biometrics. 1984; 40:1037-1048. [PubMed: 6534407]

26. Schemper M. Cox analysis of survival data with non-proportional hazard functions. The Statistician. 1992; 41:455-465. 
27. Kalbfleisch JD, Prentice RL. Estimation of the average hazard ratio. Biometrika. 1981; 68:105112.

28. Sasieni P. Maximum Weighted Partial Likelihood Estimators for the Cox Model. Journal of the American Statistical Association. 1993; 88:144-152.

29. Schemper M, Wakounig S, Heinze. The estimation of average hazard ratios by weighted Cox regression. Statistics in Medicine. 2009; 28:2473-2489. DOI: 10.1002/sim.3623. [PubMed: 19472308]

30. Barkan SE, Melnick SL, Preston-Martin S, et al. The Women's Interagency HIV Study. WIHS Collaborative Study Group. Epidemiology. 1998; 9:117-25. [PubMed: 9504278]

31. Celentano DD, Vlahov D, Cohn S, et al. Self-reported antiretroviral therapy in injection drug users. JAMA. 1998; 280:544-546. [PubMed: 9707145]

32. Celentano DD, Galai N, Sethi AK, et al. Time to initiating highly active antiretroviral therapy among HIV-infected injection drug users. AIDS. 2001; 15:1707-1715. [PubMed: 11546947]

33. Lucas GM, Cheever LW, Chaisson RE, et al. Detrimental effects of continued illicit drug use on the treatment of HIV-1 infection. J Acquir Immune Defic Syndr. 2001; 27:251-259. [PubMed: 11464144]

34. Rodriguez-Arenas MA, Jarrin I, del Amo J, et al. Delay in the initiation of HAART, poorer virological response, and higher mortality among HIV-infected injecting drug users in Spain. AIDS Res Hum Retroviruses. 2006; 22:715-723. DOI: 10.1089/aid.2006.22.715. [PubMed: 16910826]

35. Keiser O, Taffe P, Zwahlen M, et al. All cause mortality in the Swiss HIV Cohort Study from 1990 to 2001 in comparison with the Swiss population. AIDS. 2004; 18:1835-1843. [PubMed: 15316345]

36. Lau B, Gange SJ, Moore RD. Risk of non-AIDS-related mortality may exceed risk of AIDSrelated mortality among individuals enrolling into care with CD4 counts greater than 200 cells/ mm3. J Acquir Immune Defic Syndr. 2007; 44:179-187. DOI: 10.1097/01.qai. 0000247229.68246.c5. [PubMed: 17075385]

37. Leemis LM, McQueston JT. Teacher's Corner: Univariate Distribution Relationships. The American Statistician. 2008; 62:45-53. DOI: 10.1198/000313008X270448.

38. Cole SR, Hernán MA. Adjusted survival curves with inverse probability weights. Computer Methods and Programs in Biomedicine. 2004; 75:45-49. DOI: doi:10.1016/j.cmpb.2003.10.004. [PubMed: 15158046]

39. Xie J, Liu C. Adjusted Kaplan-Meier estimator and log-rank test with inverse probability of treatment weighting for survival data. Statistics in Medicine. 2005; 24:3089-110. DOI: 10.1002/ sim.2174. [PubMed: 16189810]

40. Lau B, Cole SR, Moore RD, Gange SJ. Evaluating competing adverse and beneficial outcomes using a mixture model. Statistics in Medicine. 2008; 27:4313-4327. SOI: 10.1002/sim.3293. [PubMed: 18416435]

41. Allignol A, Schumacher M, Beyersmann J. A Note on Variance Estimation of the Aalen-Johansen Estimator of the Cumulative Incidence Function in Competing Risks, with a View towards LeftTruncated Data. Biometrical Journal. 2010; 52:126-137. DOI: 10.1002/bimj.200900039. [PubMed: 20140901]

42. Jeong JH, Fine J. Direct parametric inference for the cumulative incidence function. Journal of the Royal Statistical Society: Series C (Applied Statistics). 2006; 55:187-200. DOI: 10.1111/j. 1467-9876.2006.00532.x.

43. Jeong JH, Fine J. Parametric regression on cumulative incidence function. Biostatistics. 2007; 8:184-196. DOI: 10.1093/biostatistics/kxj040. [PubMed: 16636138] 


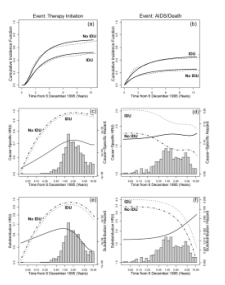

Figure 1.

The cumulative incidence function (panels a and b), cause-specific hazards (panels c and d, dotted and dash-dot-dash curves), and subdistribution hazards (panels e and $\mathrm{f}$, dotted and dash-dot-dash curves) from the multivariate mixture model applied to the HIV data in Table 1 by injection drug use (IDU) status and according to therapy initiation (left column) or AIDS/death (right column). The CIF for the multivariate mixture model from Table 1 (solid line) is shown in contrast to the non-parametric inverse probability weighted estimator (dashed line). In panels c, d, e, and f, the dotted (IDU) and dash-dot-dash (no IDU) curves correspond to the hazard (right axis), the solid line corresponds to the hazard ratio, $\operatorname{HR}(t)$ (left axis), and the histogram shows the distribution of corresponding column events. 


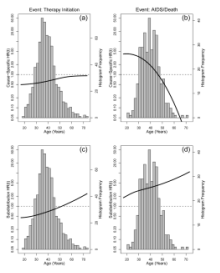

Figure 2.

The ${ }_{c s} \mathrm{HR}(t)$ (panels a and b, left axis) and the ${ }_{s d} \mathrm{HR}(t)$ (panels $\mathrm{c}$ and d, left axis) in which the time scale was age requiring left truncation in the analysis. The HRs compare those with a history of injection drug use for the initiation of therapy (left panels) prior to AIDS or death and for disease progression (right panels) prior to therapy relative to those without history of injection drug use. Curves are adjusted for nadir CD4, race, and time on study. Histogram (right axis) corresponds to the distribution of corresponding column events. 


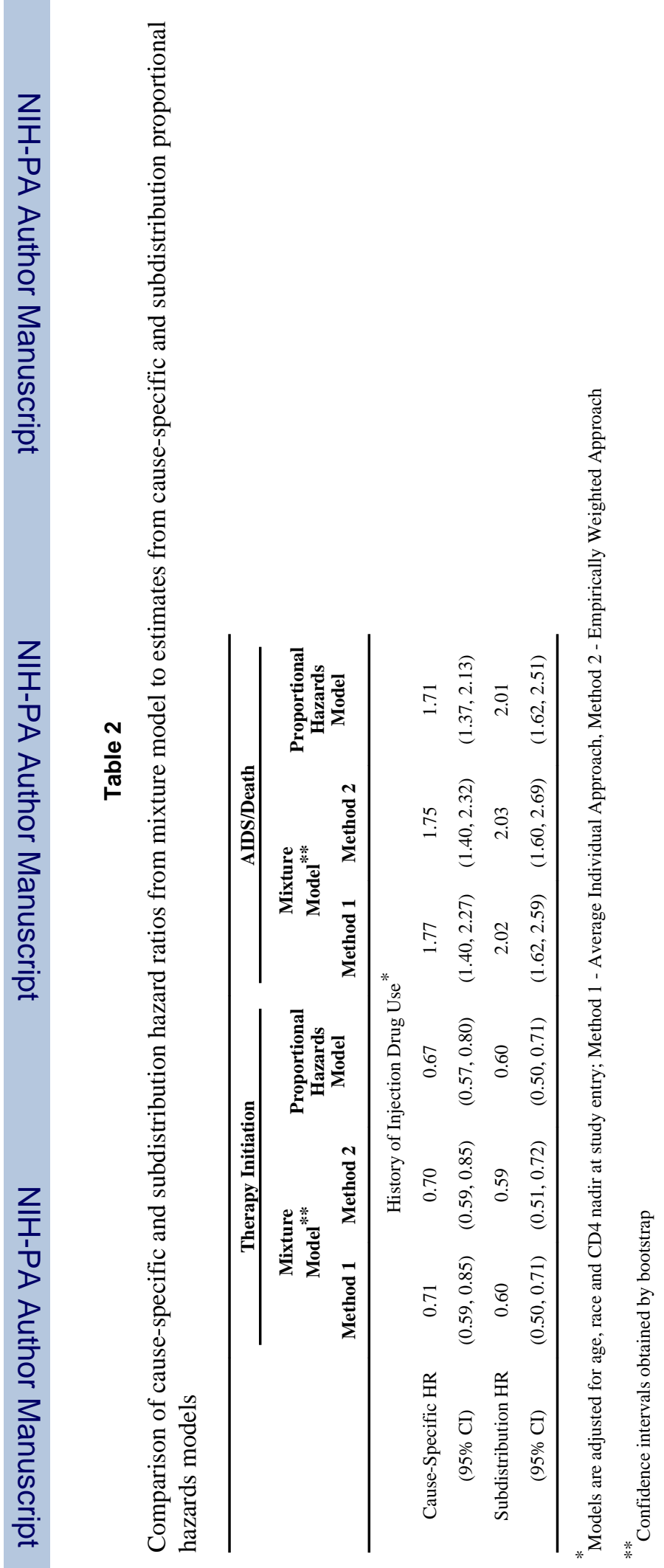

Stat Med. Author manuscript; available in PMC 2012 March 15. 